\title{
Peripheral immune profile of children with Talaromyces marneffei infections: a retrospective analysis of 21 cases
}

Qiang Zeng ${ }^{1}$, Yingkang Jin' ${ }^{1}$ Genquan Yin ${ }^{1}$, Diyuan Yang ${ }^{1}$, Wenyan $\mathrm{Li}^{2}$, Tingting Shi ${ }^{2}$, Gen Lu', Li Huang ${ }^{2 *}$ and Huifeng Fan ${ }^{1 *}$

\begin{abstract}
Background: Talaromyces marneffei (T. marneffei) is an opportunistic pathogen that infects immunodeficient children. The aim of the study is to determine the clinical features and peripheral immune state of Talaromyces marneffei (T. marneffei) infections in children for early detection and diagnosis.
\end{abstract}

Methods: We retrospectively reviewed 21 pediatric patients who were diagnosed with T. marneffei infections and were followed up in the Guangzhou Women and Children's Medical Center from January 2010 to January 2020. For each patient, we collected and analyzed clinical characteristics, peripheral immunological results, genetic tests, complications and prognosis.

(Continued on next page)

\footnotetext{
*Correspondence: huangli7525@163.com; sonny-000@163.com

${ }^{2}$ Pediatric Intensive Care Unit, Guangzhou Women and Children's Medical

Center, Guangzhou Medical University, No.9, Jinsui Road, Zhujiang New City,

Tianhe District, Guangzhou 510120, Guangdong, China

'Department of Respiratory Infection, Guangzhou Medical University, No.9, Jinsui Road, Zhujiang New City, Tianhe District, Guangzhou 510120,

Guangdong, China
}

(c) The Author(s). 2021 Open Access This article is licensed under a Creative Commons Attribution 4.0 International License, which permits use, sharing, adaptation, distribution and reproduction in any medium or format, as long as you give appropriate credit to the original author(s) and the source, provide a link to the Creative Commons licence, and indicate if changes were made. The images or other third party material in this article are included in the article's Creative Commons licence, unless indicated otherwise in a credit line to the material. If material is not included in the article's Creative Commons licence and your intended use is not permitted by statutory regulation or exceeds the permitted use, you will need to obtain permission directly from the copyright holder. To view a copy of this licence, visit http://creativecommons.org/licenses/by/4.0/. The Creative Commons Public Domain Dedication waiver (http://creativecommons.org/publicdomain/zero/1.0/) applies to the data made available in this article, unless otherwise stated in a credit line to the data. 


\begin{abstract}
(Continued from previous page)
Results: Common clinical features of the patients included fever (20/21, 95.24\%), cough (17/21, 80.95\%) and hepatomegaly (17/21, 80.95\%). Severe complications included septic shock (12/21, 57.14\%), hemophagocytic lymphohistiocytosis (HLH) (11/21, 52.38\%), acute respiratory distress syndrome (ARDS) (10/21, 47.62\%), multiple organ dysfunction syndrome (MODS) $(9 / 21,42.86 \%)$, and disseminated intravascular coagulation (DIC) (7/21, 33.33\%). Eleven children (11/21, 52.38\%) eventually died of T. marneffei infections. All patients were HIV negative. Seven cases revealed reduced antibody levels, especially lgG. Higher levels of IgE were detected in 9 cases with an obvious increase in two patients. Ten patients showed decreased complement C3 levels, some of whom had low C4 levels. Three patients displayed decreased absolute T lymphocyte counts, including the CD 4+ and CD 8+ subsets. A reduction in NK cells was present in most patients. No patient had positive nitro blue tetrazolium (NBT) test results. Nine patients were screened for common genetic mutations. Of the cases, one case had no diseasespecific gene mutation. Four children had confirmed hyperimmunoglobulin M syndrome (HIGM) with CD4OLG variation, one case had severe combined immunodeficiency (SCID), and one case had hyper-lgE syndrome (HIES). One patient was identified as having a heterozygous mutation in STAT3 gene; however, he showed no typical clinical manifestations of HIES at his age. One patient had a mutated COPA gene with uncertain pathogenic potential. Another patient was diagnosed with HIES that depended on her clinical features and the National Institutes of Health $(\mathrm{NIH})$ scoring system.

Conclusions: T. marneffei infections in HIV-negative children induced severe systemic complications and poor prognosis. Children with T. marneffei infections commonly exhibited abnormal immunoglobulin levels in peripheral blood, particularly decreased lgG or increased lgE levels, further suggesting possible underlying PIDs in these patients.
\end{abstract}

Keywords: Talaromyces marneffei, Complication, Immunity, Primary immunodeficiency diseases, Children

\section{Background}

Talaromyces (formerly Penicillium) marneffei (T. marneffei) is an opportunistic pathogen that infects immunodeficient patients in Southeast Asia as a dimorphic fungus. The fungus was first isolated from the hepatic lesions of a bamboo rat (Rhizomys sinensis) that died spontaneously from the infection in 1956 [1]. Subsequent studies showed that bamboo rats (Rhizomys $s p$. and Cannomys sp.) and soil from their burrows were important enzootic and environmental reservoirs of $T$. marneffei, respectively [2]. Generally, T. marneffei presented either as a local or disseminated infection. The symptoms and signs of disseminated T. marneffei typically include fever, weight loss, fatigue, hepatosplenomegaly, lymphadenopathy, respiratory, and gastrointestinal abnormalities, and is associated with serious complications and high death rates [3]. In adults, T. marneffei infection has been considered to be exclusively associated with acquired immunodeficiency syndrome (AIDS) caused by human immunodeficiency virus (HIV) infection [4]. Therefore, the close correlation between disease manifestation and severity with CD4+ cell count confirms the importance of cell-mediated immunity against T. marneffei in adults $[2,5]$.

The proportion of patients aged 3 months to 16 years among reported cases of talaromycosis, is approximately $6-7.5 \%$ [6-8]. Unlike the previous reports with most HIV-infected pediatric cases, recent studies showed most pediatric patients were HIV negative $[6,8]$. . At present, the research on the peripheral immune profile is less comparatively in HIV-negative children with $T$. marnef$f e i$ infections. In the previous study, the HIV-negative children had a reduction in the number of $\mathrm{T}$ lymphocytes or cellular immunity is probably the most important predisposing factor for T. marneffei infection [6]. However, the current knowledge gaps focus on the immune statuses of these children and the categories of primary immunodeficiencies (PIDs) associated with $T$. marneffei infections.

This retrospective study of 21 HIV-negative children who were infected by $T$. marneffei preliminarily assess the immune profile of these patients and to provide insights into the immunological characteristics of the disease in children.

\section{Methods}

A retrospective cohort study was conducted from January 2010 to January 2020 at Guangzhou Women and Children's Medical Center. Twenty-one children enrolled in this study who presented with culture and/or histopathologically proven infections caused by $T$. marneffei. All data were collected using a standardized form that was based entirely on the medical reports of each patient. The data included demographic information, domiciles, medical history, clinical manifestations, immunologic detection, genetic tests, complications and prognosis. 
The diagnostics of PIDs were performed based on clinical characteristics and genetic tests according to the updated classification of PIDs by the Primary Immunodeficiency Expert Committee (PID EC) of the International Union of Immunological Societies (IUIS) [9]. As noted, the National Institutes of Health (NIH) developed a clinical Hyperimmunoglobulin E Syndromes (HIES) scoring system [10], which can serve as a valuable reference for the diagnosis of HIES. The diagnostic criteria included increased $\operatorname{IgE}(>1000 \mathrm{IU} / \mathrm{mL})$ plus a weighted score of clinical features $>30$ as diagnosis of autosomal dominant HIES (AD-HIES) [11].

This study protocol was conducted in accordance with the Declaration of Helsinki and approved by the Ethics Committee of Guangzhou Women and Children's Medical Center of Guangzhou Medical University. We obtained all written informed consent from the children's parents or legal guardians for the use of their clinical and laboratory data from their medical reports.

\section{Results}

\section{Clinical characteristics}

The clinical features of the children are summarized in Table 1 and S1. There were 15 boys and 6 girls with ages ranging from 3 months to 156 months old (median age of 22 months). All patients lived in southern China, though the diagnoses were made throughout the year. The median time from the onset of symptoms to hospitalization was 20 days in our hospital (IQR:12-30 days). The most common clinical presentations of $T$. marneffei infections were fever (20/21, 95.24\%), cough $(17 / 21,80.95 \%)$ and hepatomegaly $(17 / 21,80.95 \%)$ (Table 1). Life-threatening complications during hospitalization included septic shock (12/21, 57.14\%), hemophagocytic lymphohistiocytosis (HLH) (11/21, $52.38 \%$ ), acute respiratory distress syndrome (ARDS) (10/21, 47.62\%), multiple organ dysfunction syndrome (MODS) $(9 / 21,42.86 \%)$ and disseminated intravascular coagulation (DIC) $(7 / 21,33.33 \%)$. Most patients $(19 / 21$, $90.48 \%$ ) were confirmed by blood culture, and 8 of them had also been confirmed by bone marrow culture. In addition, four cases underwent lymph node biopsy, two cases underwent airway mucosal biopsy and one case underwent skin biopsy. Two special cases confirmed by sputum culture and bronchoalveolar lavage fluid (BALF). Eleven children $(11 / 21,52.38 \%)$ eventually died from $T$. marneffei infections.

\section{Immunologic detection}

The immunologic detection and genetic tests at the time of diagnosis are shown in Table 2 and S2. All patients were HIV negative according to a serum-specific antibody test. The lymphocyte count, the content of immunoglobulin and complement, and nitro blue tetrazolium (NBT) test in peripheral blood were detected in all cases except one. Among them, seven patients had reduced immunoglobulin content (mainly IgG) (P2, 4, $10,11,12,13$ and 17). It is with great regret that 9 children were treated with intravenous immunoglobulin (IVIg) administration before the examination. There were another 9 patients who showed higher levels of IgE $(\mathrm{P} 1,5,6,7,14,15,19,20$ and 21). In particular, IgE levels were obviously increased in two cases (P7 and 21). Low complement C3 levels were found in 10 cases (P1, $2,5,6,7,8,9,12,18$ and 20), and five cases exhibited simultaneously low complement C4 levels (P1, 5, 6, 9 and 20). Of these cases, 9 were fatal. Only three cases presented decreasing $\mathrm{T}$ lymphocyte counts, including CD 4+ and CD 8+ subsets, in the results (P8, 12 and 18). P3 revealed only lower CD $8+$ subsets. Greater than half of all cases (P1, 5, 7, 8, 11, 12, 13, 14, 15, 18, 19, 20 and 21) showed markedly decreased NK cell counts. The ratio of $\mathrm{CD} 4 / \mathrm{CD} 8$ increased in 8 patients $(\mathrm{P} 1,3,4,6,7$, 9, 10 and 17). No one was positive in NBT test.

\section{Genetic tests and primary deficiencies}

For personal and economic reasons, nine patients underwent gene sequencing, three patients(P3, 9 and 19) by whole exome sequencing, six patients $(\mathrm{P} 4,10,16,17,18$ and 21) by selective PID panel testing, seven of whom were identified with specific gene variation, one patient (P9) with uncertain gene variation, and one patient (P3) without disease-specific gene mutation (Table 3). Four children (P4, 10, 16 and 17) were confirmed to have hyperimmunoglobulin $M$ syndrome (HIGM) because they identified mutations or microdeletions in the CD40LG gene. A patient (P18) was diagnosed with severe combined immunodeficiency (SCID) due to an IL2RG mutation. Another patient (P21) was diagnosed with hyper-IgE syndrome (HIES) based on his NIH score and STAT3 mutation, and the NIH score was 48 (Table S3). P19 had yet to exhibit typical clinical manifestations of HIES at his age. His NIH score was 20 although he had a heterozygous mutation in STAT3 gene. The last gene (P9) has a mutated COPA gene with uncertain pathogenic potential. Because the other 12 patients did not undergo genetic tests, their PIDs were not determined. In particular, P7 was diagnosed with HIES that depended on her clinical features and the NIH scoring system, and her score was 56 .

\section{Discussion}

Talaromyces (formerly Penicillium) marneffei(T. marnef$f e i)$ is an opportunistic pathogen that infects immunodeficient children. The aim of the study is to determine the clinical features and peripheral immune state of T. mar$n e f f e i$ infections in children for early detection and diagnosis. T. marneffei is an emerging pathogenic fungus 
Table 1 Clinical characteristics of the children with T. marneffei

\section{Clinical characteristics}

Cases $(N=21)$

\section{Demographics}

Age (months), median (IQR)

$22(15-34)$

Gender, male (\%)

15 (71.43)

Endemic area, No(\%)

$21(100.00)$

Month of diagnosis, median (IQR)

7 (4-9)

\section{Signs and Symptoms}

The interval from onset to addmission (days), median (IQR) ${ }^{\mathrm{a}}$

$20(12-30)$

Fever, No. (\%)

$20(95.24)$

Cough, No. (\%)

17 (80.95)

Hepatomegaly, No. (\%)

17 (80.95)

Lymphadenopathy, №. (\%)

15 (71.43)

Splenomegaly, No. (\%)

$12(57.14)$

Skin lesion, No. (\%)

9 (42.86)

Dyspnea, No. (\%)

$9(42.86)$

Diarrhea, No. (\%)

$6(28.57)$

Weight loss, No. (\%)

$6(28.57)$

Malnutrition, №. (\%)

4 (19.09)

Ascites, No. (\%)

Trachyphonia, No. (\%)

\section{Significant complication}

Sepsis shock, No. (\%)

$12(57.14)$

HLH, No. (\%)

$11(52.38)$

ARDS, No. (\%)

$10(47.62)$

MODS, No. (\%)

9 (42.86)

DIC, No. (\%)

7 (33.33)

\section{Specimens for diagnosis}

Blood, No(\%)

$\mathrm{BM}, \mathrm{No}(\%)$

8 (38.10)

Lymph nodes, No(\%)

4 (19.09)

Airway mucosal biopsy, No(\%)

$2(9.52)$

BALF, No(\%)

$2(9.52)$

Ascites, No(\%)

$1(4.76)$

Skin, No(\%)

$1(4.76)$

Stool, No(\%)

$1(4.76)$

\section{Antifungal therapy}

The interval from onset to antifungal therapy (days), median (IQR)

$25(17.5-31.5)$

Total, No(\%)

Amphotericin B, No(\%)

Voriconazole, $\mathrm{No}(\%)$

Itraconazole, No(\%)

Fluconazole, No(\%)

$5(23.81)$

Caspofungin, $\mathrm{No}(\%)$

$3(14.29)$

\section{Outcomes}


Table 1 Clinical characteristics of the children with T. marneffei (Continued)

\begin{tabular}{l}
\hline Clinical characteristics \\
\hline Mortality, No. (\%)
\end{tabular}

that can cause fatal systemic mycosis in immunocompromised hosts and occurs mostly in humid tropical climate regions, including the south and southwest regions of China [12]. In adult, T. marneffei infection occurs mainly in AIDS patients, where it is a severe, deep mycosis with high mortality [4]. In contrast to adults, subjects with PIDs are more susceptible in children according to previous reports $[6,8]$. Furthermore, the recognition of immune status by clinical observation is important to treat and prevent $T$. marneffei infections in children and to facilitate the diagnosis and reporting of PIDs. We retrospectively analyzed the immunity status of 21 children with $T$. marneffei infection over this decade, including the immunoglobulin pattern (IgG, IgA, IgM, and $\operatorname{IgE}$ ) and enumeration of lymphocyte subpopulations (T-, B-, and NK-cells) in peripheral blood. We found that abnormal immunoglobulin findings were considerable in HIV-negative children with T. marneffei infection, mainly in those with decreased IgG or increased IgE.
In our study, we summarized 21 documented pediatric cases of proven T. marneffei infection in southern China. Most cases presented with fever, weight loss, swollen lymph nodes, generalized lymphadenopathy, and hepatomegaly. In many aspects, the clinical manifestations of pediatric patients are different from those of adult patients $[6,8,13]$. For example, respiratory system involvement was also observed in most cases, but skin lesions were unusual in pediatric patients in the present study. A high frequency $(70-80 \%)$ of skin lesions has been reported in adults with T. marneffei infection [14]. Nonspecific clinical manifestations are a potential cause of misdiagnosis for $T$. marneffei infection. T. marneffei infection is a severe disease that can lead to high mortality rates of greater than $50 \%$ in children in previous reports [6]. Of these cases, 11 were fatal, and all fatal cases in our study died of serious complications, such as HLH, septic shock, MODS, DIC and ARDS. It is noteworthy that the incidence of HLH in pediatric patients was significantly higher than that in

Table 2 Immunoassays findings of the children with T. marneffei

\begin{tabular}{|c|c|}
\hline Immunoassays findings & Cases $(N=21)$ \\
\hline \multicolumn{2}{|l|}{ Immunoglobulin $^{a}$} \\
\hline Pan-hypogammaglobulinemia (lgG, IgA and IgM), No(\%) & $1(4.76)$ \\
\hline IgG decrease, No(\%) & $7(33.33)$ \\
\hline IgA decrease, No(\%) & $5(23.81)$ \\
\hline IgM decrease, No(\%) & $1(4.76)$ \\
\hline IgM increase, $\mathrm{No}(\%)$ & $6(28.57)$ \\
\hline lgE increase, No(\%) & $9(42.86)$ \\
\hline \multicolumn{2}{|l|}{ Complements } \\
\hline C3 decrease, No(\%) & $10(47.62)$ \\
\hline C4 decrease, No(\%) & $5(23.81)$ \\
\hline \multicolumn{2}{|l|}{ Lymphocyte counts } \\
\hline T lymphocyte counts decrease (CD4+ and CD8+), No(\%) & $3(14.28)$ \\
\hline CD4+ subsets decrease, $\mathrm{No}(\%)$ & $3(14.28)$ \\
\hline CD8+ subsets decrease, $\mathrm{No}(\%)$ & $4(19.09)$ \\
\hline The ratio of CD4/CD8 increase, $\mathrm{No}(\%)$ & $8(38.10)$ \\
\hline NK cells decrease, No(\%) & $13(61.90)$ \\
\hline \multicolumn{2}{|l|}{ NBT tests } \\
\hline Normal, No(\%) & $21(100.00)$ \\
\hline \multicolumn{2}{|l|}{ HIV detection } \\
\hline Negative, $\mathrm{No}(\%)$ & $21(100.00)$ \\
\hline
\end{tabular}

Abbreviation: Ig immunoglobulin, NK cells natural killer cells, NBT test nitro blue tetrazolium test, HIV human Immunodeficiency virus

${ }^{\mathrm{a}}$ Nine children were treated with intravenous immunoglobulin (IVIg) administration before the examination 
Table 3 Genetic detection and primary immunodeficiencies of the children with T. marneffei

\begin{tabular}{llllll}
\hline Patient & Gene & Genetic variation & Nucleotide variation & PIDs & Gene testing \\
\hline 3 & Not Found & Not Found & Not Found & Not Determined & Whole exome gene sequencing \\
4 & CD4OLG & Hemizygote & C.424_436del & HIGM & PID genes panel \\
7 & Not done & - & - & HIES & - \\
9 & COPA & Heterozygous & C.2437G $>$ T & Not Determined & Whole exome gene sequencing \\
10 & CD4OLG & Microdeletion & $>132 \mathrm{~kb}$ & HIGM & PID genes panel \\
16 & CD4OLG & Microdeletion & C.1978 + 1G $>$ A & HIGM & PID genes panel \\
17 & CD4OLG & Hemizygote & C.598A $>$ T & HIGM & PID genes panel \\
18 & IL2RG & Hemizygote & C.185G $>$ A & SCID & PID genes panel \\
19 & STAT3 & Heterozygous & C.1679_1681del & Not Determined & Whole exome gene sequencing \\
21 & STAT3 & Heterozygous & C.A1593T & HIES & PID genes panel \\
\hline
\end{tabular}

Abrreviation: PIDs primary immunodeficiencies, HIGM hyperimmunoglobulin M syndrome, HIES hyper-lgE syndrome, SCID severe combined immunodeficiency, NIH National Institutes of Health

${ }^{a}$ The patient was diagnosed with HIES based on her clinical features and the NIH scoring system

${ }^{\mathrm{b}}$ The NIH score of this patient remained substandard to confirmed HIES at his age

adults, possibly because many of our children had delayed treatment due to early misdiagnosis. Another major cause of complications and high mortality in HIV-negative patients with $T$. marneffei infection may be abnormal host immune function.

In the present study, all of these children were HIV negative, but they almost had abnormal immune parameters at the time of diagnosis, especially in the immunoglobulin contents, including decreased levels of IgG or increased levels of IgE in peripheral blood. However, in only three cases, the number of peripheral blood lymphocytes significantly decreased. Previous studies have suggested that a reduction in the number of $\mathrm{T}$ lymphocytes or cellular immunity is probably the most important predisposing factor for adult T. marneffei infection [6, 7]. Serum antibody levels were increased in HIV-negative adults in one study [15]. Unlike adults, the decreased levels of IgG in peripheral blood were found in 7 cases. There were 9 cases with increased IgE, two of them had markedly elevated IgE levels. Taking into account that 9 cases were treated by IVIg prior to the immune detection being performed, which certainly influenced immunoglobulin levels in peripheral blood. Even so, the common immunoglobulin changes in children with T. marneffei infection are lower IgG or higher IgE in peripheral blood. That is, pediatricians should be alert to T. marneffei infection in children when peripheral immunoglobulin changes.

On the other hand, abnormal immunity may occur secondary to serious T. marneffei infection. Previous studies focused less on complement levels. In our study, approximately half of all patients displayed decreased complement levels, especially C3. It should be noted that of the 11 deaths of our children, 9 cases of complement dramatically declined. The complement system has a determinant role in defense against infections [16], so the reduction in complement is probably also an important index of children with severe T. marneffei infection. In addition, reduced NK cell counts were identified in most patients. NK cells are the prototype innate lymphoid cells endowed with potent cytolytic functions that provide host defense against microbial infection [17]. The reduction in NK cells may be due to HLH secondary to severe T. marneffei infection. Further studies are necessary to evaluate immunologic parameters to explore the influence of body immunity from severe T. marneffei infection.

Abnormal immunological findings might suggest underlying PIDs in HIV-negative children with T. marneffei infection. This point has been reported in children with various forms of immune-related underlying diseases and PIDs $[7,18,19]$. As a further study of T. marneffei infection, we performed nine genetic tests of underlying PIDs in recent years. The results showed that four cases were identified as HIGM with mutations or microdeletion of the CD4OLG gene. There were two patients with mutations in STAT3 gene, one of whom was diagnosed with HIES in combination with typical clinical features. We have not diagnosed HIES in another child now, considering many clinical signs did not appear at his age (1 year old). His NIH score was only 20, but it was not completely excluded. A hemizygous mutation of IL2RG was identified in one patient diagnosed with SCID. The immunoassay results of the patient displayed reduced numbers of lymphocyte subsets along with remarkable declines in immunoglobulin levels. This finding is consistent with IL2RG mutation for definitive diagnosis. One patient had a mutated COPA gene with uncertain pathogenic potential. Patients with COPA mutations typically have normal numbers and percentages of lymphocytes and lymphocyte subsets along with unremarkable immunoglobulin levels and intact production of specific antibodies [20]. However, the child had remarkedly decreased immunoglobulin with normal numbers of lymphocytes. The exact mechanism 
by which COPA gene mutation causes T. marneffei infection is currently unknown. Although no genetic testing was performed on 12 patients, some of them showed degrees of abnormal immunity in peripheral blood. Of note is patient 7. She was diagnosed with HIES based on her clinical features and the NIH scoring system, but she did not receive genetic tests. In addition, P2, P11, P12 and P13 were accompanied by a decrease in IgG levels, and P12 coincided with obviously reduced counts of lymphocyte subsets. This finding might suggest that these patients could have had potentially underlying immunodeficiencies that were not identified or that their poor prognosis was linked to a more systemic impairment in immunity.

The study still has some limitations that should be considered. This is a retrospective single-center analysis, and some earlier HIV-negative children with T. marneffei did not receive genetic tests; hence, the proportion of such cases with underlying PIDs is unknown. In addition, serum neutralizing anti-IFN- $\gamma$ autoantibodies has not yet been carried out in our hospital, it is with great regret that our patients failed to get tested. Furthermore, anti-IFN- $\gamma$ autoantibodies associated immunodeficiency is partly due to inherited mutations in IFN- $\gamma$-signaling-associated factors, however we such mutations were not detected in our small cohort. We will pay more attention to the disease and perform the testing in the future. Nevertheless, this study may provide a valuable reference for immunity monitoring in children with $T$. marneffei infection.

\section{Conclusions}

T. marneffei infections in HIV-negative children involve many systemic complications, high mortality and poor prognosis. There were obvious immunoglobulin abnormalities in these children with severe T. marneffei infections in peripheral blood, especially in decreased IgG or increased IgE, suggesting possibly underlying PIDs.

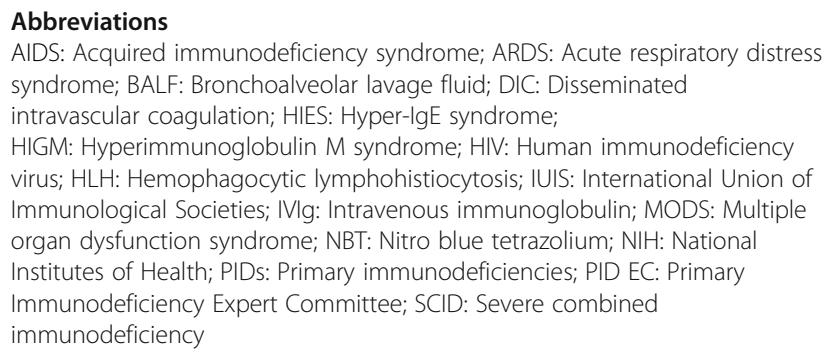

\section{Supplementary Information}

The online version contains supplementary material available at https://doi. org/10.1186/s12879-021-05978-z.
Additional file 1.
Additional file 2.
Additional file 3.

\section{Acknowledgements}

We are very appreciative to the children and their families.

\section{Authors' contributions}

QZ and LH conceived and designed the study. QZ and HFF analyzed data and wrote the paper. GL and GQY analyzed data and modified the paper. YKJ, DYY, TTS and WYL collected patients' clinical data. All authors read and approved the final manuscript.

\section{Funding}

Not applicable.

\section{Availability of data and materials}

The datasets generated and/or analyzed during the current study are not publicly available due individual privacy of patients could be compromised, but are available from the corresponding author on reasonable request.

\section{Declarations}

\section{Ethics approval and consent to participate}

This study protocol was conducted in accordance with the Declaration of Helsinki and approved by the Ethics Committee of Guangzhou Women and Children's Medical Center of Guangzhou Medical University. Written informed consents were signed during hospitalization. The data used in this study was anonymised before its use.

\section{Consent for publication}

Not applicable.

\section{Competing interests}

The authors declare that they have no competing interests to disclose.

Received: 14 December 2020 Accepted: 8 March 2021

Published online: 20 March 2021

\section{References}

1. Capponi M, Segretain G, Sureau P. Penicillosis from Rhizomys sinensis. Bull Soc Pathol Exot Filiales. 1956;49(3):418-21.

2. Vanittanakom N, Cooper CR Jr, Fisher MC, Sirisanthana T. Penicillium marneffei infection and recent advances in the epidemiology and molecular biology aspects. Clin Microbiol Rev. 2006;19(1):95-110.

3. Cao C, Xi L, Chaturvedi V. Talaromycosis (penicilliosis) due to talaromyces (penicillium) marneffei: insights into the clinical trends of a major fungal disease 60 years after the discovery of the pathogen. Mycopathologia. 2019; 184(6):709-20.

4. Limper AH, Adenis A, Le T, Harrison TS. Fungal infections in HIV/AIDS. Lancet Infect Dis. 2017;17(11):e334-43.

5. Supparatpinyo K, Chiewchanvit S, Hirunsri P, Uthammachai C, Nelson KE, Sirisanthana T. Penicillium marneffei infection in patients infected with human immunodeficiency virus. Clin Infect Dis. 1992;14(4):871-4.

6. Guo J, Li BK, Li TM, Wei FL, Fu YJ, Zheng YQ, Pan KS, Huang CY, Cao CW. Characteristics and prognosis of talaromyces marneffei infection in non-HIVinfected children in southern China. Mycopathologia. 2019;184(6):735-45.

7. Lee PP, Chan KW, Lee TL, Ho MH, Chen XY, Li CH, Chu KM, Zeng HS, Lau YL. Penicilliosis in children without HIV infection--are they immunodeficient? Clin Infect Dis. 2012;54(2):e8-e19.

8. Fan H, Huang L, Jin Y, Chen C, Lu G, Zhang D, Jiang W. Study of Penicillium marneffei infection in pediatric patients without human immunodeficiency virus infection in china. Pediatr Allergy Immunol Pulmonol. 2017;30(1):53-9.

9. Picard C, Al-Herz W, Bousfiha A, Casanova JL, Chatila T, Conley ME, Cunningham-Rundles C, Etzioni A, Holland SM, Klein C, et al. Primary immunodeficiency diseases: an update on the classification from the International Union of Immunological Societies Expert Committee for primary immunodeficiency. J Clin Immunol. 2015;35(8):696-726. https://doi. org/10.1007/s10875-015-0201-1.

10. Grimbacher B, Schaffer AA, Holland SM, Davis J, Gallin JI, Malech HL, Atkinson TP, Belohradsky BH, Buckley RH, Cossu F, et al. Genetic linkage of hyper-lgE syndrome to chromosome 4. Am J Hum Genet. 1999;65(3):735-44.

11. Woellner C, Gertz EM, Schaffer AA, Lagos M, Perro M, Glocker EO, Pietrogrande MC, Cossu F, Franco JL, Matamoros N, et al. Mutations in 
STAT3 and diagnostic guidelines for hyper-lgE syndrome. J Allergy Clin Immunol. 2010;125(2):424-432 e428.

12. Hu Y, Zhang J, Li X, Yang Y, Zhang Y, Ma J, Xi L. Penicillium marneffei infection: an emerging disease in mainland China. Mycopathologia. 2013; 175(1-2):57-67.

13. Zeng W, Qiu Y, Lu D, Zhang J, Zhong X, Liu G. A Retrospective analysis of 7 human immunodeficiency virus-negative infants infected by Penicillium marneffei. Medicine. 2015;94(34):e1439.

14. Ranjana KH, Priyokumar K, Singh TJ, Gupta Ch C, Sharmila L, Singh PN Chakrabarti A. Disseminated Penicillium marneffei infection among HIVinfected patients in Manipur state, India. J Infect. 2002;45(4):268-71.

15. Wong SS, Wong KH, Hui WT, Lee SS, Lo JY, Cao L, Yuen KY. Differences in clinical and laboratory diagnostic characteristics of penicilliosis marneffei in human immunodeficiency virus (HIV)- and non-HIV-infected patients. J Clin Microbiol. 2001;39(12):4535-40

16. Conigliaro P, Triggianese P, Ballanti E, Perricone C, Perricone R, Chimenti MS. Complement, infection, and autoimmunity. Curr Opin Rheumatol. 2019; 31(5):532-41.

17. Morvan MG, Lanier LL. NK cells and cancer: you can teach innate cells new tricks. Nat Rev Cancer. 2016;16(1):7-19.

18. Han XJ, Su DH, Yi JY, Zou YW, Shi YL. A literature review of blooddisseminated P. marneffei infection and a case study of this infection in an HIV-negative child with comorbid eosinophilia. Mycopathologia. 2019;184(1): 129-39.

19. Fan H, Huang L, Yang D, Lin Y, Lu G, Xie Y, Yu J, Zhang D. Pediatric hyperimmunoglobulin $E$ syndrome: a case series of 4 children in China. Medicine. 2018:97(14):e0215.

20. Vece TJ, Watkin LB, Nicholas S, Canter D, Braun MC, Guillerman RP, Eldin KW Bertolet G, McKinley S, de Guzman M, et al. Copa syndrome: a novel autosomal dominant immune dysregulatory disease. J Clin Immunol. 2016; 36(4):377-87.

\section{Publisher's Note}

Springer Nature remains neutral with regard to jurisdictional claims in published maps and institutional affiliations.

Ready to submit your research? Choose BMC and benefit from:

- fast, convenient online submission

- thorough peer review by experienced researchers in your field

- rapid publication on acceptance

- support for research data, including large and complex data types

- gold Open Access which fosters wider collaboration and increased citations

- maximum visibility for your research: over $100 \mathrm{M}$ website views per year

At BMC, research is always in progress.

Learn more biomedcentral.com/submissions 